\title{
A hybrid intelligent recognition system for the early detection of strokes
}

\author{
José R. Villar ${ }^{\mathrm{a}, *}$, Camelia Chira ${ }^{\mathrm{b}}$, Javier Sedano ${ }^{\mathrm{c}}$, Silvia González ${ }^{\mathrm{c}}$ and José M. Trejo ${ }^{\mathrm{d}}$ \\ ${ }^{a}$ Computer Science Department, University of Oviedo, Independencia, Oviedo, Spain \\ ${ }^{\mathrm{b}}$ Technical University of Cluj-Napoca, Cluj-Napoca, Romania \\ ' Instituto Tecnológico de Castilla y León, López Bravo, Burgos, Spain \\ ${ }^{\mathrm{d}}$ Neurology Department, Hospital de Burgos, Burgos, Spain
}

\begin{abstract}
The increasing prevalence of wearable sensors and low-cost mobile devices have prompted the development of systems for automated diagnosis. Here we focus on models and algorithms for the early detection of strokes that are implanted in a wearable device that generates warning alarms and automatically connects to e-health services, ensuring timely interventions at the onset of a stroke. The proposed approach employs two wearable devices to monitor movement data that involve two main stages: Human Activity Recognition (HAR) and alarm generation. Two different HAR methods capable of classifying current human activity are developed and compared: one uses genetic fuzzy finite-state machines, and the other relies on Time Series (TS) analysis. Furthermore, an algorithm using Symbolic Aggregate approXimation (SAX) TS representation is proposed for alarm generation purposes, which is triggered by the detection of anomalous movements. The proposed methods are evaluated using realistic data gathered from healthy individuals. A discussion of topics related to the learning issues involved in these techniques is included. It is worth mentioning that the proposed algorithms can be easily transferred to embedded systems and can benefit from reduced learning costs.
\end{abstract}

Keywords: Early stroke detection, human activity recognition, ambient assisted living, time series analysis, SAX

\section{Introduction}

Over recent years, the focus of Artificial Intelligence applications in medicine has ranged from braincomputer interfaces for human assistance [24]3540, 44] and devices for diagnostic purposes [3- 5 [ 17 |28], to specific utilities for information extraction [7/23] and efficient microarray analysis [11]. Even early mortality prediction is a research focus [21]. One of the most promising fields is computer-based diagnosis [34], particularly early stroke detection.

Stroke diagnosis is a very challenging problem that has only been partly addressed [1932|37] Although diagnostic methods may vary with age [37], there are clear symptoms of a stroke episode, described by the

* Corresponding author: José R. Villar, Computer Science Department, University of Oviedo, ETSIMO, c/Independencia 13, Oviedo 33004, Spain. E-mail: villarjose@uniovi.es.
FAST (Face drooping, Arm paralysis, Speech difficulties, Time to act [19]) informative campaign for early diagnosis [31|32]. By far the most remarkable symptom of a stroke is the loss of voluntary movement in one or more of the limbs.

In all cases, a patient suffering from the onset of a stroke needs a trained care assistant close by to recognize the symptoms; in many stroke situations, an isolated individual would be unable to request help alone. Interestingly, there are factors that increase the risks of stroke onsets, among them cholesterol [49], smoking, and the intake of a certain amount of alcohol [33]. A very up-to-date definition and review of strokes may be found in [38], while [43] contains an impressive account of a personal stroke episode by a doctor.

Cerebral infarction explains around $85 \%$ of all strokes [1]. There is no approved treatment for the remaining $15 \%$ of all cerebral hemorrhages, although thrombolytic drug treatment can counter cerebral in- 
farction, by clearing the thrombus that may occlude the cerebral arteries. If successful, cerebral tissue and its function will recover, although the speed of treatment is crucial. In the first one-and-a-half hours (the so-called "golden hour"), one out of every three patients treated in this way will fully recover.

Frequently, there are several different signs and symptoms, although these are usually accompanied by paralysis, which is responsible for most temporary and permanent disabilities. Indeed, hemiplegia is the main sequel [12] of a stroke with grave consequences for the patient and indirectly for the carer. The hand is usually more severely affected than the leg. Arm paralysis is in fact so common in stroke patients that it appears in well-known scales, such as the Prehospital Cincinnati Stroke Scale. Even with a complex, drawn out rehabilitation process, recovery is not always completely achieved [13].

The movements of a person suffering a stroke episode are usually described as asymmetrical: one side moves while the other remains rather still [43]. The main symptom is collapse while standing still, walking, or even when seated. Hand paralysis is nonindividual and may be monitored by a movementdetection device. These devices can learn the main patterns of normal behavior of each type of activity. Consequently, detecting either asymmetrical movements or deviations in these normal patterns would allow early detection of strokes.

Hybrid Artificial Intelligent Systems provide useful methods for the development of tools to diagnose human illnesses and human behavior [7 29|41|50]. A method based on Genetic Fuzzy Finite State Machines (GFFSM) was recently proposed for Human Activity Recognition $(H A R)$ [6], and applied in the early stroke diagnosis [48].

In this paper, the response is taken one step further by presenting an alternative to the HAR module: early stroke diagnosis is addressed using wearable devices $3 \mathrm{D}$ accelerometers $(3 D A C C)$-equipped with alarms for stroke onset recognition. The aim is to develop a noninvasive and cheap device that can detect abnormal behavior in the individual; repetition of such movements should set off the alarm, alerting health workers and, whenever necessary, emergency health care services, [22] and dosing the thrombolytic drug within the golden hour.

The approach in this work is presented in two steps: a HAR stage and a Stroke Onset Detection stage. The former is responsible for classifying current Human Activity ( $c H A)$ and the latter for evaluating the abnor- mality of the current movements once the cHA classification is given. A bracelet that incorporates 3DACC was developed, through a complete analysis and evaluation of the proposed algorithm, and was then tested in the experimentation stage.

The organization of this paper is as follows. The next section summarizes the basic knowledge for early stroke diagnosis. Then, in Section 3 , the proposed approach is detailed. The experimentation and discussion on the results are then included in Section 4, The main conclusions of the research appear at the end of the paper.

\section{Design decisions related to wearable devices for early stroke diagnosis}

We will focus on the stroke risk population, which includes adults above 56 years of age. Within this group, we will try to measure and to identify a selection of the most suitable activity subset covering, as far as possible, the relevant everyday activities that are valid for stroke diagnosis, including Resting, Walking and Small Movements.

Ambulation is a well studied topic [29] and there are enormous differences in movements depending on age: the older the individual, the smaller the degree of movement. Thus, the evaluation of frequency related patterns is not suitable as it varies with age and individuals.

While Walking is conceptually clear [16], the remaining activities need further explanation [27]. Resting refers to situations in which the individual is either seated or lying down and is totally inactive, for instance when sleeping, resting or having a nap. Small Movements refers to situations in which the individual is seated, lying down or standing up while performing any low-level activity. It is worth noting that the active individual can simultaneously perform several different tasks. For instance, an individual might be walking while snacking or repeating a pattern while speaking. In this study, the identification of gestures is not addressed.

Finally, it is worth mentioning the special case of falling. Whenever an elderly person suffers a fall, it may result in a fracture -typically- of the hip or pelvis. In this case, the individual might be conscious but cannot ask for help. Conversely, the fall might produce concussion, due perhaps to a stroke episode, requiring immediate assistance, which would therefore require the alarm to be set off. Furthermore, it would of inter- 
est to categorize relative wrist positions, so as to define relatively abnormal positions that would assist in the alarm generation process.

Apart from these movement-related issues, some restrictions need to be considered, so that the approach would not only be valid but also realistically useful. Firstly, the device should be wearable and conveniently fitted, without discomfort, in the daily life of the individual wearer. Secondly, it should be affordable. Thirdly, it should be user friendly and easy to configure: any learning and tuning processes for optimal adaption to individual needs, if required, should be easy to follow.

All these restrictions lead to several design decisions. The number of sensors should be kept as low as possible, to reduce both cost and the size of the device. In this study, only two tri-axial accelerometer sensors are proposed, each one in a bracelet worn on the wrist. This design is based on the idea that the upper limbs are more affected than the lower limbs in stroke situations and that the lack of movement is more generally observed in the hands for any human activity. Moreover, the available techniques and all the implemented models should be valid for deployment in micro-controlled devices. The premise "Keep It Simple" needs to underlie any decision and its implementation.

\section{Proposed approach for stroke episode recognition and stroke onset early detection}

This study proposes a two-block approach for early stroke diagnosis: a HAR method to determine the cHA, allowing us to discriminate between human activities, and an alarm generation stage where a pattern recognition method is used to classify movements (as normal or otherwise) from the cHA detected by the HAR device. The data from the 3DACC feeds directly into a pre-processing stage, which is needed as each block requires a different set of features and acceleration transformations. Figure 1 depicts the flowchart of the blocks; the remainder of the section is devoted to the presentation of all details behind the proposed approach.

The data from the 3DACC sensors (one on each wrist) are used for detecting the cHA as well as for detecting abnormal behavior of the individual. Consequently, the stroke alarm state is activated whenever there is a lack of movement or whenever the movements suggest that a fall might have occurred.

Placement of the sensors on the wrist has previously been confirmed as a valid approach in this type of sit-

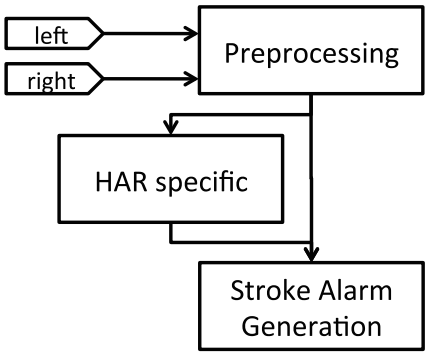

Fig. 1. The block diagram for the whole approach. Left and Right represent the tri-axial accelerometer data from each wrist. After the pre-processing, the HAR takes place and then the alarm generation is updated.

uation [36]. Even though some reports show that the wrists might be the worst location for obtaining good HAR results [52], the need to evaluate abnormal behavior means that this placement is more suitable, as opposed to the torso or the legs, for instance, as they barely reflect any change when the individual is engaged in low-motion activities such as reading.

We therefore need to estimate the current activity of the individual and then whether the lack of motion is due to paralysis or a fall. In a previous study, a HAR method making use of GFFSM [6] was adapted in [15] and enhanced [48]. However, there is a limit on the number of activities that can be considered, and finding valid alternatives might enhance the overall HAR and assist with the correct definition of the aforementioned limit.

This topic is one of the main goals of this research and is studied in depth in the next subsection. The current research goes one step further with a different HAR method and a proposal for the alarm generation stage. The goal is to keep these modules sufficiently simple to allow their implementation in microcontrolled devices. Moreover, the key data, such as the cHA for each wrist and the stroke alarm state should where necessary be sent to an e-Health system.

This section is divided into three subsections, each dealing with an overview of the previous approach, the novel HAR method and the alarm generation stage.

\subsection{Human activity recognition using GFFSM}

A two-stage HAR method has recently been proposed in [48]: on the one hand, a Feature Selection $(F S)$ stage reduces the input domain dimensionality; on the other, GFFSM modeling generates the classifier. The cross validation considered the crossing of individuals rather than the crossing of data samples. Furthermore, the FS included two stages: a filtering 
FS stage followed by a wrapper FS phase. The final GFFSM modeling trained the Fuzzy Finite State Machine with different learning strategies [2/42]: several approaches were compared, ranging from Pittsburgh fixed-size rule set tuning to boosting Michigan learning.

The cross-validation shuffles the data from the individuals that will be used for training and for validation. In this way, all the Time Series (TS) from a single individual are considered either for training or for validation purposes, according to the cross-validation shuffling that is performed.

FS filtering is a very interesting voting algorithm for the fusion of the results of different Principal Component Analyses (PCA, [14|29 51|). Whenever PCA is applied, the rollback of the $M$ most relevant transformations -up to $95 \%$ of representation- is performed. To do so, the transformations are sorted according to their percentage of representation. Let us consider that a transformation is located at position $p$. This transformation is assigned a rank $(M-p+1) / M$, and the features involved in its computation receive the vote of the rank times the percentage of representation times the corresponding coefficient. In this way, each feature is voted on the basis of the PCA results by aggregation of the marks from all the transformations in which the feature is involved.

Two main ballots were performed: one for the whole training data set and a second one fold by fold. In the former case, the whole training data set is aggregated for all the individuals, then PCA is performed, and the features are then voted. In the latter case, PCA is independently performed for each fold; so different voting results are obtained. Finally, the approval-voting scheme is applied [8] by aggregating the votes from each fold, using the mean of the votes. The voting scheme is also applied to the results from each ballot, using the same weight. As a result, each feature in the original space gets a vote from the different PCA transformations, which is used for ranking and filtering.

The second FS stage, as mentioned above, is a wrapper FS. In this case, the FS method is an adapted version [45-47] of the Steady State Genetic Algorithm FS [10]. The wrapper makes use of a genetic algorithm to learn a GFFSM; a relaxed set of parameters is used in this learning, in order to reduce the computational cost. Consequently, the output of this FS is a feature subset used for developing the GFFSM classifier with a suitable set of parameters to optimize the model; however, the GFFSM will need further training before its performance is acceptable.
The last stage of the proposal referred to in [48] deals with the learning of the final GFFSM models once the feature subset has been chosen. The original GFFSM -where the 3DACC were placed on the center of the individual's back- has been adapted to the current problem. Furthermore, different learning strategies, varying from Pittsburgh learning to the boosting of Michigan fuzzy rules, were all analyzed and compared. The most promising method found so far was GFFSM learned with Boosting with the Single Winner Inference algorithm [39].

This approach appeared to fit the HAR requirements perfectly, which is one of the steps in the current study for stroke recognition. Nevertheless, it could be said that the higher the number of activities the lower the performance of the HAR method. Consequently, the activity set should be limited to only those activities that may be interesting for the stroke recognition.

\subsection{SAX-based human activity recognition}

The limitation regarding the number of activities led us to analyze different approaches that would eventually be implemented in embedded devices. Interestingly, the analysis of different TS representations [30] showed the simplicity and potential use of Symbolic Aggregate approXimation (SAX) [25] in this context.

Using 3DACC means that the measurements of raw data from the sensors should be decomposed into gravity acceleration $(G)$ and body acceleration $(B A)$, resulting from human movement. Using a lowpass and a highpass filter, respectively, we can obtain both accelerations with its three components per axis. The value of BA may be computed as

$$
B A_{i}=\sqrt{\left(b_{i}^{x}\right)^{2}+\left(b_{i}^{y}\right)^{2}+\left(b_{i}^{z}\right)^{2}},
$$

with the following three components: $\left(b_{i}^{x}, b_{i}^{y}, b_{i}^{z}\right)$. The capacity of the BA to discriminate between different human gestures is documented in [51].

Let $m B A_{\{L, R\}}$ and $s B A_{\{L, R\}}$ be the mean and standard deviation of the movements during the daily living activities of the individual, both calculated by means of an initial test. The sub-index $L$ and $R$ stand for the left and the right hand, respectively. These values will be used for normalization of the TS windows.

The algorithm proposed for HAR is included in Fig. 2; for the sake of brevity, it is shown rather schematically. This algorithm makes use of SAX, a well-known TS representation technique [25]. The main idea is that for each of the focused activities, a set 


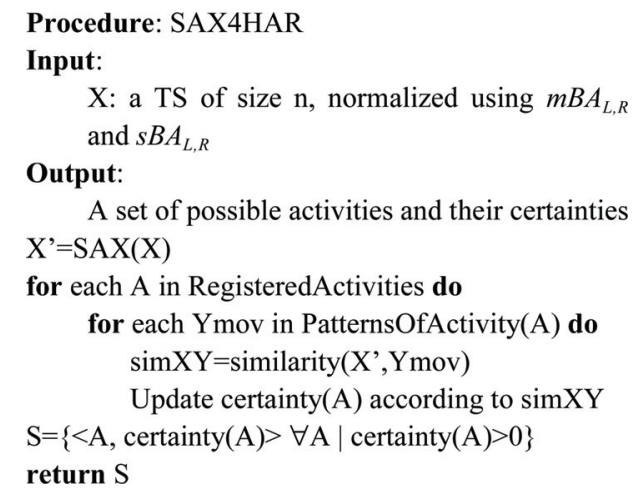

Fig. 2. Algorithm for HAR using the SAX TS representation.

of SAX patterns that are fairly usual when performing the activity should be identified. These patterns should be as general as possible, in the sense that if they are common to a wide variety of individuals the results are more generalized. However, it is enough to carry out these activities for a short period of time and then extract them using I-Nearest Neighbor or similar algorithms [26].

Once the parameters detailed above are set, the problem is solved as classifying the cHA based on activities with a similar associated pattern. The aggregation of similarities can be any t-conorm, such as the Lukasiewicz t-conorm -the bounded addition-.

Some issues need further study, for example learning the membership functions and their tuning to the focused individual, as well as the method for measuring similarities between TS representation. Interestingly enough, the computational cost and resources needed for implementing this algorithm are kept rather small, which is one of the main enhancements of this technique. The last part of the algorithm is related to the point at which the alarm is generated at the onset of a stroke or after a fall.

\subsection{Alarm generation following stroke onset and falls}

With regard to alarm generation, the study proposed in [47], where an algorithm for alarm generation based on the SAX TS representation is proposed, is worth mentioning. Besides, the approach presented in [9] was considered both for abnormal movement detection and for detecting falls.

The TS from both bracelets are used in this case, and the state of resting should be previously detected, on an independent basis for each hand. In the case of a resting state, the $\mathrm{BA}$ is normalized using the $\mu_{R B A \_} h$ and $\sigma_{R B A_{-} h}$, the mean and standard deviation of the sig-

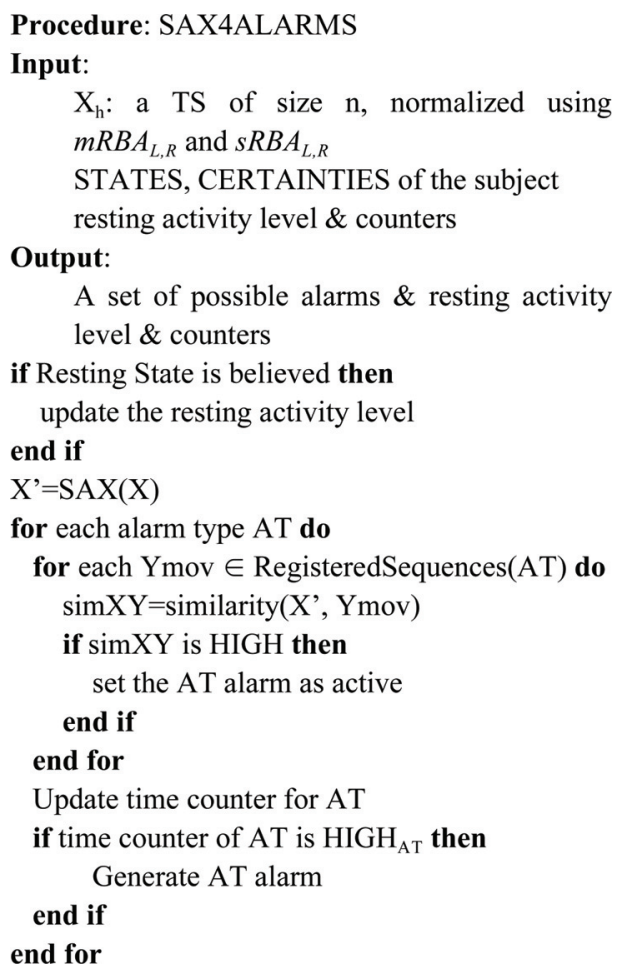

Fig. 3. Simplified alarm generation algorithm. This algorithm is the responsible of detecting any alarm condition and of generating the corresponding alarms when needed.

nal of the BA when the individual is resting; the $\mathrm{h}$ subindex is related with the -left or right- hand. These values should be calculated in an initial test.

The SAX4ALARMS algorithm shown in Fig. 3 briefly outlines the proposed approach. In its current state, the algorithm is focused on alarm generation in the RESTING state, because this is the most common scenario at the onset of a stroke. However, the structure would be the same, even if the whole set of alarms were considered: just by introducing more alarms and their corresponding patterns and thresholds.

The algorithm works as follows. Firstly, the cHA is estimated -by means of a HAR method, the SAX4HAR for instance-; the time spent in each state is also updated. Whenever the individual is in a RESTING state, the current resting amount of movement level is determined.

Three different resting activity levels have been considered: when the individual has just begun a RESTING state (short rests, with the individual carrying out some low activity tasks like reading), when doing nothing -for instance, if the individual is having a nap, then the activity level is clearly low- and when the individual is sleeping- which means that the activity level is highly reduced over a longer period of time. 
The similarity of the signal from each hand is compared with the typical sequences of inactivity for each case. The certainty of setting off the corresponding alarms for either partial or total paralysis, or for fall detection, increases with the patterns that are found. For instance, considering the case of the individual in a RESTING state, when one hand shows no activity and the other hand shows some, then that might suggest partial paralysis. Conversely, a rather low activity level in both hands might characterize total paralysis. Each alarm has a minimum waiting time in the same alarm state, with specific time periods for each alarm.

In this study, health experts have provided the following time thresholds. The first threshold (THI) is calculated using the scenario in which an upper limb is considered to be affected by paralysis on the basis of the current activity of the individual. If the individual is found RESTING, on the basis of the movements of one hand, and is found not to be RESTING, on the basis of the other hand, for more than 6 minutes, then the partial paralysis alarm is set off. Thus TH1 is the number of samples according to the sampling rate for which the individual should remain in this state before activating the partial paralysis alarm.

The second threshold $T H 2$ is related with the RESTING state for both hands, so if one of the hands is performing active resting -i.e., it is moving slightly to try to reach an object- and the other does not perform any movement for more than 6 minutes, then the partial paralysis alarm is once again activated. Again, TH2 is the corresponding number of samples in this scenario for this alarm set up.

Finally, the third threshold, TH3, is concerned with RESTING for long periods, such as when having a nap or when asleep at night. In this case, if a hand does not perform any movement for periods of time longer than 14 minutes, then the alarm for partial paralysis is triggered. TH3 also includes the corresponding number of samples for this period.

When the partial paralysis alarms are set off for both hands, then the total paralysis alarm is also triggered. As stated in the case of HAR, the set of typical sequences or patterns for each alarm type and hand are learned in a previous training phase with the individual, as the patterns are rather specific to each person.

This proposal and the associated algorithms are still under design and evaluation; it is worth mentioning that the whole approach has a reduced computational cost that can easily be implemented in embedded solutions.

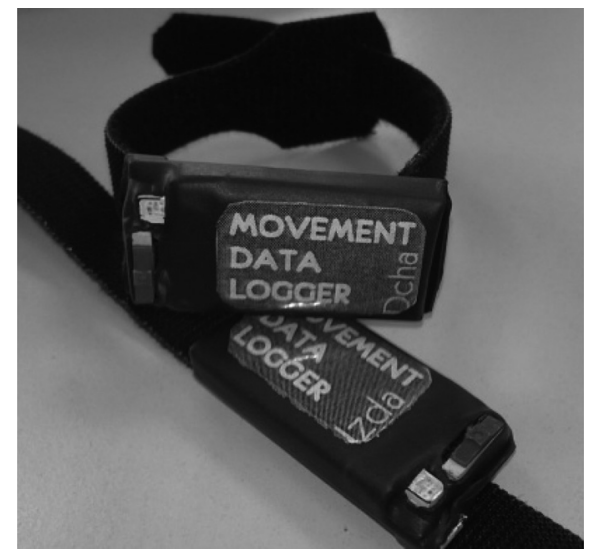

Fig. 4. The tri-axial bracelets used in this study, one for each hand.

\section{Experiments and results}

Several tests have been carried out as follows: i) an evaluation and comparison of SAX-based HAR methods; and, ii) the evaluation of the SAX-based method for stroke alarm generation, in order to evaluate the approach presented in this study. This section deals with each of these steps: the first subsection describes the details of data collection and cross-validation; the second subsection reports the test results and discusses the above-mentioned points.

\subsection{Materials and experimental methods}

In this study, a pair of data logging wrist bracelets, with a sampling frequency of $16 \mathrm{~Hz}$, was developed (see Fig. 4). Each bracelet, which is identified for the left and the right hand, includes a tri-axial accelerometer and a USB port. Using these bracelets, an individual completes the different tests with the corresponding repetitions for statistical purposes; after which the data is segmented.

Two different tests were considered in this study: i) a HAR test for evaluating the SAX-based HAR method; and, ii) a test for validating the SAX algorithm for stroke alarm generation. Each of these tests needs specific data sets that were obtained using the above bracelets.

A well-known stroke rehabilitation test (for short, SRT [18]) was chosen for gathering the data sets concerning the HAR comparison. Two individuals carried out 10 runs of the SRT and a TS was obtained from each run. The individuals were of a different sex and age, both outside the focus population. The first individual was ambidextrous, while the second individual was right handed. 
In the SRT, an individual remains seated for a period of time $\mathrm{T} 1$, then stands up in the same position for a period of time $\mathrm{T} 2$, and then walks along a line on the floor, coming back to the original standing position and remaining there for $\mathrm{T} 2$ seconds, and finally sits down and rests for $\mathrm{T} 1$. The line on the floor is a 10 -meter straight line leading to another 10-meter straight line at an angle of 45 degrees from the former.

All the data were manually segmented and classified according to the individual activity, in the same way as was proposed in the GFFSM in the original work [2]. For computing the features, a sliding window 10 samples wide and one sample shift were used. In this way, known states were classified with complete certainty, while transitions between actions were assigned with imprecise data, e.g., 0.7/SEATED + 0.3/STANDING. This means that up to $10 \mathrm{TS}$ were gathered and segmented for each individual. Finally, the segmented data for each run was considered a data set, so up to 10 different data sets were available for training and testing.

Typically, the HAR studies in the literature make use of a 10-fold cross-validation. If a 10-fold crossvalidation on the data sets from of the 10 repetitions of the SRT test are carried out, 10 folds would be generated, each one containing 9 data sets for training purposes and one data set for the final validation. However, in our opinion, this cross-validation method is neither suitable for HAR method evaluation, nor for comparitive purposes: the generality of the model is not significantly well tested.

Besides, the SAX-based HAR method requires no parameter-based training; only the calculation of some statistical estimators taken from the data. In this study, we advance a more challenging cross-validation method: to do so, we propose a 10 -fold cross validation where only one single data set of those from the 10 repetitions of the SRT test will be used for the calculation of the statistics, while the remaining data sets will be used for the validation of the results of the model.

In fact, the higher the ratio between the size of the test and the training data sets, the higher the expected error; however, this cross-validation scheme would eventually lead us to evaluate the generalization of the model. Using this scheme, a better resemblance of real deployment of the device and the models is obtained, as many completely unknown TS are given to the model. The lower the training parameters of a HAR method, the easier its deployment.

Moreover, data sets for evaluating the SAX-based stroke alarm generation algorithm have also been gathered. In this case, the individual was asked to sleep
Table 1

MAE results for HAR using the SAX4HAR for the first subject

\begin{tabular}{cccccc}
\hline & \multicolumn{2}{c}{ Left hand } & & \multicolumn{2}{c}{ Right hand } \\
\cline { 2 - 3 } \cline { 5 - 6 } Fold & Train & Test & & Train & Test \\
\hline 1 & 0.1045 & 0.1374 & & 0.1312 & 0.2018 \\
2 & 0.0406 & 0.1597 & & 0.1621 & 0.2271 \\
3 & 0.1377 & 0.1849 & & 0.1765 & 0.2274 \\
4 & 0.0710 & 0.1184 & & 0.0956 & 0.1558 \\
5 & 0.2476 & 0.1216 & & 0.2396 & 0.1474 \\
6 & 0.0548 & 0.0625 & & 0.0818 & 0.1104 \\
7 & 0.0416 & 0.0713 & & 0.0711 & 0.1268 \\
8 & 0.0418 & 0.0602 & & 0.0563 & 0.1038 \\
9 & 0.0403 & 0.0636 & & 0.0459 & 0.1086 \\
10 & 0.0492 & 0.0591 & & 0.3450 & 0.0708 \\
Mean & 0.0829 & 0.1039 & & 0.1405 & 0.1480 \\
Median & 0.0520 & 0.0949 & & 0.1134 & 0.1370 \\
Std & 0.0664 & 0.0467 & & 0.0941 & 0.0546 \\
\hline
\end{tabular}

wearing the bracelets for ten nights; the bracelets were turned on once the individual was in the bed, and the data was gathered. In this scenario, no segmentation is needed because the individual is resting. The same cross-validation scheme detailed above was used: the TS from one run was used for learning the statistic parameters and the remaining TS were used for studying the evolution of the algorithm.

\subsection{Evaluation of the HAR method}

The Mean Absolute Error (MAE, see Eq. (1) was used for evaluating each individual when learning a GFFSM model. Thus, the MAE will also be used for evaluating the SAX4HAR method. For computing the MAE, $T_{i}$ is the number of examples in the data set $i, N$ is the number of available data sets, and $s_{i}[t]$ and $s_{i}^{*}[t]$ are the degree of generation state $q_{i}[t]$ and the expected degree of generation, respectively, both at time step $t$.

$$
M A E=\frac{1}{N} \sum_{i=1}^{N} \frac{1}{T_{i}} \sum_{t=1}^{T_{i}} a b s\left(s_{i}[t]-s_{i}^{*}[t]\right)
$$

The results obtained for the SAX4HAR method are presented in Tables 1 to 6 . The results shown in Tables 1 and 2 are concerned with the MAE from the cross-validation folds. The results yield errors of around 10\%; however, these statistics give no clear idea of how the TS are classified.

From Tables 3 to 6, the corresponding confusion matrices for each hand are included. From these results, in spite of the training based on data from a single data set, the SAX4HAR correctly classifies the majority of samples in the RESTING and the WALKING states. However, the results for the STANDING state were not 
Table 2

MAE results for HAR using the SAX4HAR for the second subject

\begin{tabular}{cccccc}
\hline & \multicolumn{2}{c}{ Left hand } & & \multicolumn{2}{c}{ Right hand } \\
\cline { 2 - 3 } \cline { 5 - 5 } Fold & Train & Test & & Train & Test \\
\hline 1 & 0.1026 & 0.1515 & & 0.1095 & 0.1527 \\
2 & 0.1874 & 0.1412 & & 0.1897 & 0.1402 \\
3 & 0.1742 & 0.1398 & & 0.1678 & 0.1369 \\
4 & 0.1738 & 0.1436 & & 0.1812 & 0.1434 \\
5 & 0.1697 & 0.1417 & & 0.1694 & 0.1400 \\
6 & 0.1453 & 0.1371 & & 0.1564 & 0.1340 \\
7 & 0.1106 & 0.1481 & & 0.1161 & 0.1448 \\
8 & 0.1210 & 0.1471 & & 0.1167 & 0.1500 \\
9 & 0.1257 & 0.1321 & & 0.1110 & 0.1304 \\
10 & 0.1027 & 0.1516 & & 0.1094 & 0.1527 \\
Mean & 0.1413 & 0.1434 & & 0.1427 & 0.1425 \\
Median & 0.1355 & 0.1426 & & 0.1366 & 0.1418 \\
Std & 0.0328 & 0.0063 & & 0.0330 & 0.0077 \\
\hline
\end{tabular}

Table 3

Confusion matrix for the first subject's left hand

\begin{tabular}{|c|c|c|c|c|}
\hline \multirow{5}{*}{ 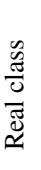 } & & \multicolumn{3}{|c|}{ Obtained class } \\
\hline & & Standing & Resting & Walking \\
\hline & Standing & 560 & 2927 & 288 \\
\hline & Resting & 4901 & 30661 & 4758 \\
\hline & Walking & 8155 & 1268 & 32625 \\
\hline
\end{tabular}

Table 4

Confusion matrix for the first subject's right hand

\begin{tabular}{|c|c|c|c|c|}
\hline \multirow{5}{*}{ 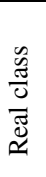 } & & \multicolumn{3}{|c|}{ Obtained class } \\
\hline & & Standing & Resting & Walking \\
\hline & Standing & 616 & 3270 & 344 \\
\hline & Resting & 5261 & 33769 & 4944 \\
\hline & Walking & 8243 & 1381 & 27680 \\
\hline
\end{tabular}

Table 5

Confusion matrix for the second subject's left hand

\begin{tabular}{|c|c|c|c|c|}
\hline \multirow{5}{*}{ 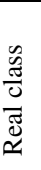 } & & \multicolumn{3}{|c|}{ Obtained class } \\
\hline & & Standing & Resting & Walking \\
\hline & Standing & 672 & 17108 & 3091 \\
\hline & Resting & 2316 & 36617 & 253 \\
\hline & Walking & 430 & 2805 & 30830 \\
\hline
\end{tabular}

Table 6

Confusion matrix for the second subject's right hand

\begin{tabular}{|c|c|c|c|c|}
\hline \multirow{5}{*}{ 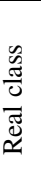 } & & \multicolumn{3}{|c|}{ Obtained class } \\
\hline & & Standing & Resting & Walking \\
\hline & Standing & 1261 & 18106 & 1369 \\
\hline & Resting & 2266 & 36186 & 194 \\
\hline & Walking & 838 & 1973 & 31011 \\
\hline
\end{tabular}

so valid. This may be due to the SRT, which sets a long standing still position for the individual for a relatively long time, which is unusual in human behavior. Moreover, this period of standing still that is set by the SRT establishes that the individuals should keep calm, with almost no movement at all: hence the SAX4HAR clas-
Table 7

Experiment parameters for each of the GFFSM approaches

\begin{tabular}{lcl}
\hline Parameter & wGFFSM & bGFFSM \\
\hline Generations & 300 & $300+10 /$ rule \\
Population size & 100 & 50 \\
Elite pop. size & 1 & 40 \\
Subpop. size & - & 10 \\
Crossover prob. & 0.8 & 1 \\
Mutation prob. & 0.02 & 0.1 \\
Interchange prob. & - & 0.01 \\
Crossover BLX $\alpha$ & 0.3 & 0.3 \\
No. of rules & 8 & Up to 6 \\
Generations w/o progress & 50 & $100+10 /$ rule \\
Logitboost $\alpha$ & - & 0.75 \\
Maximum number of iterations & - & 4 \\
\hline
\end{tabular}

Table 8

MAE results for the wGFFSM and the second subject

\begin{tabular}{cccccc}
\hline & \multicolumn{2}{c}{ Left hand } & & \multicolumn{2}{c}{ Right hand } \\
\cline { 2 - 3 } \cline { 5 - 6 } Fold & Train & Test & & Train & Test \\
\hline 1 & 0.0000 & 0.3561 & & 0.0055 & 0.2929 \\
2 & 0.0028 & 0.0511 & & 0.0014 & 0.0935 \\
3 & 0.0043 & 0.2636 & & 0.0023 & 0.0095 \\
4 & 0.0000 & 0.0214 & & 0.0002 & 0.1722 \\
5 & 0.0075 & 0.0190 & & 0.0003 & 0.1530 \\
6 & 0.0000 & 0.0608 & & 0.0008 & 0.0181 \\
7 & 0.0005 & 0.0233 & & 0.0020 & 0.0967 \\
8 & 0.0000 & 0.0099 & & 0.0005 & 0.0369 \\
9 & 0.0090 & 0.0979 & & 0.0000 & 0.0159 \\
10 & 0.0000 & 0.1273 & & 0.0004 & 0.0349 \\
Mean & 0.0024 & 0.1041 & & 0.0014 & 0.0921 \\
Median & 0.0002 & 0.0560 & & 0.0007 & 0.0652 \\
Std & 0.0034 & 0.1175 & & 0.0017 & 0.0906 \\
\hline
\end{tabular}

sifies a high percentage of the samples incorrectly at this stage. Furthermore, the SRT test produces a relatively smaller percentage of samples in this state, so the set of SAX sequences is not properly extracted. Further research is needed in order to make the SAX4HAR algorithm more robust in this type of scenario.

In addition, the SAX4HAR approach has been compared with a well-known HAR method: the GFFSM proposed in [2], adapted in [15] and outperformed in [48]. The two versions of this latter work will be used in the comparison; namely the enhanced Pittsburgh GFFSM ( $w$ GFFSM) and the Michigan-style classifier using boosting and single winner inference (bGFFSM). All the methods were trained using data from one SRT run and tested with the remaining folds, in order to compare the current solution with these two approaches. It is worth noticing that training a GFFSM with this cross-validation scheme will penalize the outcome of the methods, although this scheme would better resemble a real scenario -where the users can not be reasonably asked to perform long and cumbersome training tests-. 
Table 9

MAE results for the bGFFSM and the second subject

\begin{tabular}{cccccc}
\hline & \multicolumn{2}{c}{ Left hand } & & \multicolumn{2}{c}{ Right hand } \\
\cline { 2 - 3 } \cline { 5 - 6 } Fold & Train & Test & & Train & Test \\
\hline 1 & 0.1610 & 0.2839 & & 0.0598 & 0.2881 \\
2 & 0.0362 & 0.3127 & & 0.0202 & 0.1764 \\
3 & 0.3124 & 0.0947 & & 0.2623 & 0.3686 \\
4 & 0.3060 & 0.0860 & & 0.3089 & 0.0827 \\
5 & 0.3198 & 0.3191 & & 0.4625 & 0.1404 \\
6 & 0.3593 & 0.2443 & & 0.2997 & 0.0388 \\
7 & 0.0985 & 0.2650 & & 0.3130 & 0.0508 \\
8 & 0.3103 & 0.2924 & & 0.2274 & 0.2841 \\
9 & 0.0624 & 0.2951 & & 0.2939 & 0.2929 \\
10 & 0.3049 & 0.1967 & & 0.3043 & 0.1078 \\
Mean & 0.2271 & 0.2390 & & 0.2552 & 0.1832 \\
Median & 0.3055 & 0.2745 & & 0.2968 & 0.1584 \\
Std & $\mathbf{0 . 1 2 3 4}$ & $\mathbf{0 . 0 8 6 0}$ & $\mathbf{0 . 1 2 8 8}$ & $\mathbf{0 . 1 1 7 3}$ \\
\hline
\end{tabular}

Table 10

Confusion matrix for the second subject's left hand using the wGFFSM; 9011 samples are classified as Transitions

\begin{tabular}{|c|c|c|c|c|}
\hline \multirow{5}{*}{$\begin{array}{l}\tilde{\tilde{J}} \\
\frac{\tilde{U}}{\tilde{D}} \\
\tilde{\sim}\end{array}$} & & \multicolumn{3}{|c|}{ Obtained class } \\
\hline & & Standing & Standing & Walking \\
\hline & Standing & 33117 & 3858 & 1296 \\
\hline & Resting & 1178 & 18487 & 1066 \\
\hline & Walking & 2530 & 1175 & 23628 \\
\hline
\end{tabular}

Table 11

Confusion matrix for the second subject's right hand using the wGFFSM; 15479 samples are classified as transitions

\begin{tabular}{|c|c|c|c|c|}
\hline \multirow{5}{*}{ 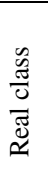 } & & \multicolumn{3}{|c|}{ Obtained class } \\
\hline & & Standing & Resting & Walking \\
\hline & Standing & 26116 & 1758 & 3929 \\
\hline & Resting & 486 & 20267 & 760 \\
\hline & Walking & 1 & 1590 & 24960 \\
\hline
\end{tabular}

The parameters used for training the wGFFSM and the bGFFSM are given in Table 7. The second individual results for both methods are shown in Tables 8 to 11, and Figs 5 and 6 for comparison purposes. The results for the best GFFSM individual found so far are reported for both hands in Tables 8 (wGFFSM) and 9 (bGFFSM). The interested reader can compare these results with those obtained for the SAX4HAR (see Tables 1 and 2). Clearly, the wGFFSM results were slightly better than the SAX4HAR results; however, the variability in their performance is a compromise and can easily be seen in Figs 5 and 6. The span of the results for SAX4HAR is really narrow, while the wGFFSM has a wider spread of results. This means that SAX4HAR is more robust with respect to the training data; while the performance of the wGFFSM is bound by the same upper limit as the SAX-based method. Furthermore, the bGFFSM has the worst performance of the two comparative methods.

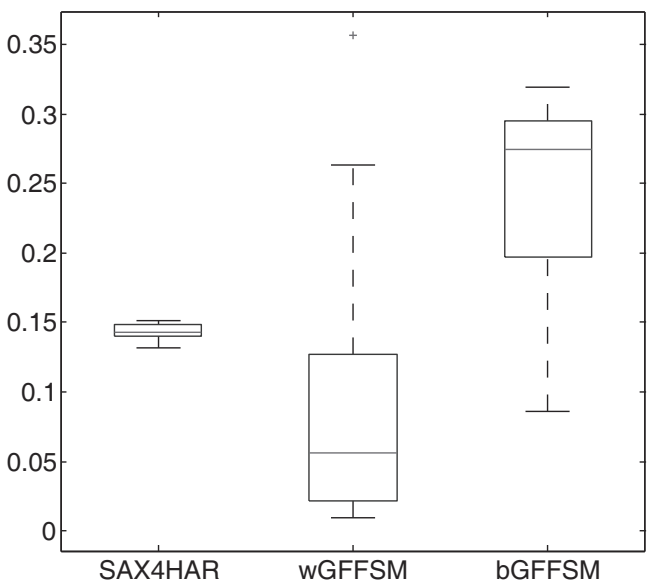

Fig. 5. Boxplot of the MAE results for the left hand. The wGFFSM has better error values, although the errors are highly dependent on the data. The SAX4HAR is more robust against the data set variability.

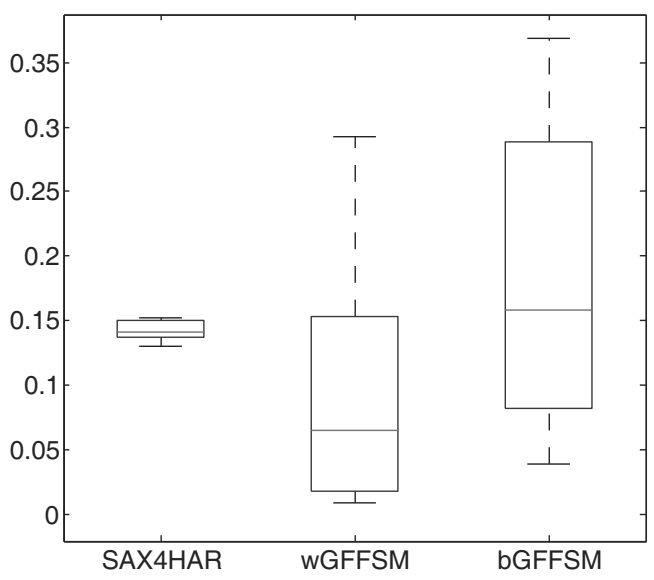

Fig. 6. Boxplot of the MAE results for the right hand. The same conclusions as for the left hand (Fig. 5) can be found in this boxplot, although in this case there is no clear best method.

The wGFFSM confusion matrices are shown in Tables 10 and 11 for the left and right hands, respectively. These confusion matrices cannot be compared with those of the SAX4HAR, because the transitions are not classified by the GFFSM: the confusion matrixes seem to be better than those of the SAX4HAR, because these unclassified samples are not included. Nevertheless, if the percentage of corrected classified samples is computed, it can be seen that SAX4HAR is rather similar or even better than wGFFSM, except for the STANDING class.

This fact is quite interesting, as one of the main claims of this study is that the approach needs almost no training, only the mean and standard deviation of 
the different states. The reduced training feature comes from the fact that the smaller the number of actions for setting up a device, the better its acceptance by the consumer. Consequently, the experimentation results show SAX4HAR as a very promising solution.

To conclude, we can state that i) the SAX4HAR is a valid HAR recognition method with a reduced computational cost; ii) there is a need for improving the set of sequences for characterizing the different states, particularly the STANDING state; and iii) the number of states might be increased provided that computation time and resources for the different TS comparison remain low.

\subsection{Evaluating the alarm generation module}

Once the HAR is considered solved, the focus shifts to the alarm generation patterns in response to the onset of a stroke. This section is devoted to the evaluation of the alarm generation algorithm.

To do so, data from the older individual while asleep over ten different nights was gathered. The SAX4HAR algorithm, together with the alarm generation module, was run using the same cross-validation scheme as for HAR. Thus, the whole approach has, in the first place, to classify the state as RESTING, and, in the second, to generate the alarms according to the given thresholds.

Two sets of thresholds will be given to test this alarm generation routine; one with approximate values established by experts, another with a set of extremely reduced threshold values, to simulate possible stroke onsets, testing the validity of the alarm generation rules. This decreased set of thresholds is simply to give a clear picture of alarm generation; the thresholds should be evaluated with individuals in realistic tests.

As stated above, the time thresholds TH1, TH2 and TH3 were fixed to the corresponding number of samples for a period of 6 minutes, 6 minutes, and 14 minutes, respectively. However, whenever the alarms were set off in order to test the alarm generation algorithm, the thresholds were computed using seconds instead of minutes.

Two periods of the night for an individual were presented, each 40 minutes long, in order to show the results: scenario 1 at the beginning of the night, and scenario 2 in the middle of the night. The SAX4ALARMS algorithm was run twice: with the normal set of thresholds and with the reduced set.

Results from the stroke alarm generation experimentation are shown in Figs 7 (scenario 1) and 8 (scenario 2). In both figures, the upper part shows the

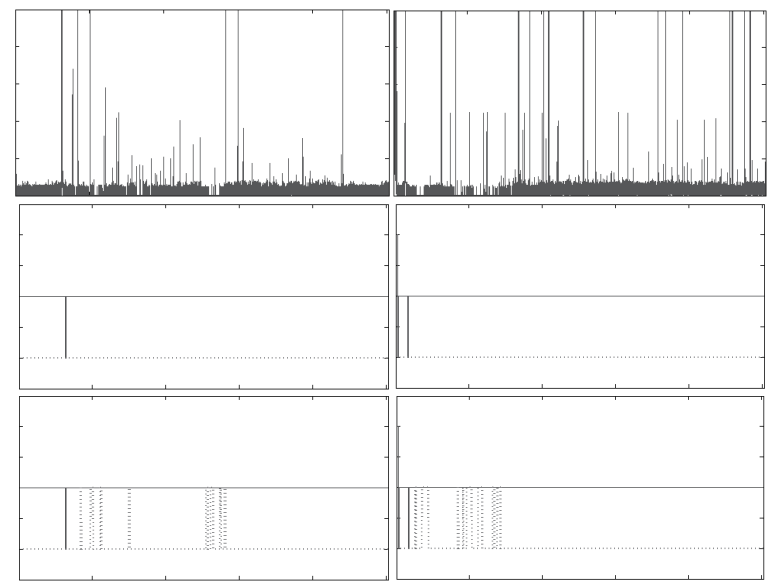

Fig. 7. Results for stroke alarm generation: scenario 1, a nighttime period when the individual is asleep. The upper part shows the BA for the left and right hands on their corresponding sides -the $y$-axis range is 0 to 0.1 , showing very small movements-. The central part depicts the estimated state -almost always fixed to Resting- and the alarm for arm paralysis -the dotted line- which does not go off; on the left and on the right hand side, respectively. The lower part includes the same variables, but with highly underestimated time thresholds so that the alarms go off.

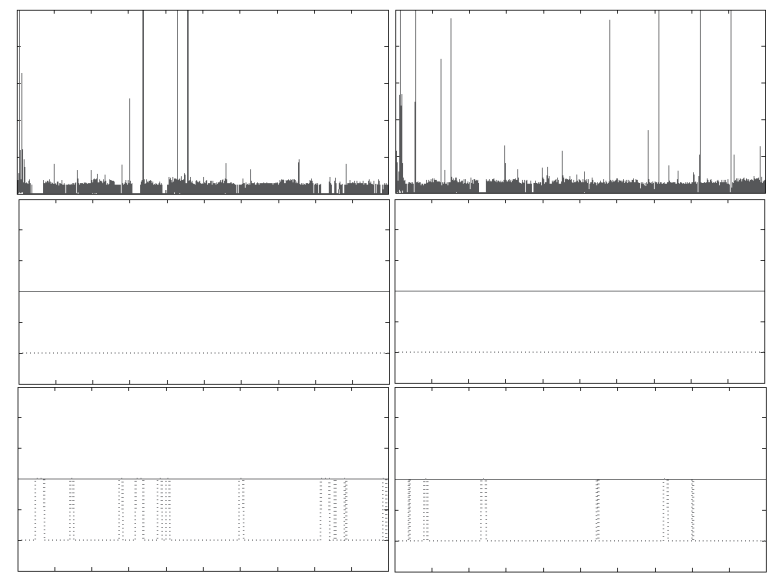

Fig. 8. Results for the stroke alarm generation, in scenario 2. The upper part includes the BA values; the central and upper part shows the SAX4ALARMS algorithm results with the time thresholds fixed by the experts -central part- and the highly underestimated time thresholds -lower part-. As for scenario 1, the alarms went off in those periods where no activity was detected.

BA when the individual is asleep at nighttime. The $y$-axis maximum values are $0.1 \mathrm{~g}$, which means that there was no activity at all. The central and lower parts of these figures show the results of evaluating the SAX4ALARMS algorithm on the TS: the lower part corresponds to running the algorithms with highly reduced time thresholds, so that the alarms will be set off. The dotted line that is shown in these central and 
lower parts is the alarm generation state. In the central part, it can be seen that the alarm was never set off. Nevertheless, several alarms were set off in the lower part.

\subsection{Discussion of results}

Some differences in the fluctuation of the BA for the two scenarios were noted, as at the beginning of the night there is more activity than when the individual is deeper asleep. Nevertheless, the TS was classified as RESTING in both scenarios as the output during the test for the SAX4HAR algorithm. The SAX4HAR algorithm may be enough for specific activities, such as RESTING, which is of high relevance in this stroke episode onset recognition, because in the most serious cases the individual can collapse. Furthermore, the simplicity of this algorithm is one of the main advantages.

It is worth noticing that the movements of each hand are completely independent, so the need for studying the partial paralysis appears to be a good decision.

Besides, when the thresholds were reduced by a matter of seconds the alarms were clearly set off (see the lower parts of both Figs 7 and 8). Although the algorithm for setting off the alarms is fairly simple, it seems to perfectly match the requirements for normal individuals. Nonetheless, it has to be tested on individuals from the target population. Furthermore, the different thresholds need to be validated and, perhaps, new alarm rules might be proposed for different stroke types as well as heuristics. For instance, if a fall is detected and then no activity is detected except RESTING and the alarms are set off, then the individually almost certainly has a serious problem.

The time thresholds will limit the time response of the algorithm, that is, longer thresholds will induce bigger time gaps between the onset of the stroke and the alarm generation. Additionally, the algorithms proposed in this study can easily be transferred into embedded systems.

It can quite rightly be said that the SAX4ALARMS algorithm includes no intelligent models. The inclusion of thresholds and these types of rules is not a new proposal and has been validated in certain situations; for instance, one of the best known examples concerns the detection of falls [8]. Often, the simpler approach is better. Applying the simplicity principle as expressed in the form of Ockham's Razor is certainly an effective means of countering high computational models [20]. Nonetheless, it is clear that a compromise usually has to be struck between simplicity and highly complex methods.

It is important to stress the way in which this approach should be deployed. The impact of smartphones in society provides new opportunities for progress in biomedicine, mainly in cases of diagnosis and patient logging. In this case, the approach should be completed as follows. On the one hand, the individual should be given two bracelets, one for each hand. These bracelets should make use of low energy communications, such as Blue Tooth Low Energy. Preprocessing and communications should be analyzed to balance energy consumption. These devices should be connected to the individual's Smart watch, responsible for sending the information to the web services and for local signaling, to attract the attention of bystanders in a position to provide assistance. Web-based services should manage the assignment of medical services.

The solution presented in this study, once completed and validated, will potentially reduce the time gap between stroke episode onset and delivery of thrombolytic medication by means of ITC. Finally, it should be said that all issues concerned with the distribution of the decision-making process are beyond the scope of this study, despite the obvious interest in their discussion.

\section{Conclusions}

This study has concerned itself with ambient assisted living issues for the generation of alarms in cases of stroke onset, which could allow timely delivery of thrombolytic medication, to mitigate the long-term effects of these attacks.

A solution has been presented, based on wearing two bracelets that include 3D accelerometers that work with intelligent algorithms: i) for classifying human activity; and; ii) for setting off the alarms when the individual's behavior does not show a symmetrical amount of movement. A specific bracelet has been developed and a complete analysis of both algorithms has been carried out in the experimentation stage.

Some interesting results of this study are that, concerning HAR, a simple algorithm can outperform more complex models in terms of results and computational costs. The algorithms proposed in this study may easily be transferred into embedded systems. Moreover, the alarm generation module, although still in need of further experimentation with the target population, appears to be suitable for setting off alarms in case of an asymmetrical amount of movement. 
Quite clearly, experimentation with the target population calls for special agreements with each individual and various national associations, among other reasons to protect data privacy; these types of agreements can de drawn out and would require suitable funding. Ongoing and future work focuses on these two directions to continue to enrich and to expand this research.

\section{Acknowledgments}

This research has been supported through Spanish Ministry of Science and Innovation projects TIN201124302 and TIN2014-56967-R, Junta de Castilla y León projects BIO/BU09/14, SACYL 2013 GRS/822/A/13, CCTT/10/BU/0002 and CCTT/10/LE/0001, and Fundación Universidad de Oviedo project FUO-EM-34013 (IE13-112).

\section{References}

[1] H.P. Adams, G. del Zoppo, M.J. Alberts, D.L. Bhatt, L. Brass, A. Furlan, R.L. Grubb, R.T. Higashida, E.C. Jauch, C. Kidwell, P.D. Lyden, L.B. Morgenstern, A.I. Qureshi, R.H. Rosenwasser, P.A. Scott and E.F.M. Wijdicks, Guidelines for the early management of adults with ischemic stroke, stroke 38 (2007), 1655-1711.

[2] H. Adeli and S.L. Hung, Machine learning-neural networks, genetic algorithms and fuzzy sets, John Wiley and Sons, New York, 1995.

[3] M. Ahmadlou, H. Adeli and A. Adeli, Fractality and a wavelet-chaos-neural network methodology for EEG-based diagnosis of autistic spectrum disorder, Journal of Clinical Neurophysiology 27(5) (2010), 328-333.

[4] M. Ahmadlou, H. Adeli and A. Adeli, Improved visibility graph fractality with application for diagnosis of autism spectrum disorder, Physica A: Statistical Mechanics and its Applications 391(20) (2012), 4720-4726.

[5] M. Ahmadlou, H. Adeli and A. Adeli, Fractality analysis of frontal brain in major depressive disorder, International Journal of Psychophysiology 85 (2012), 206-211.

[6] A. Álvarez-Álvarez, G. Triviño and O. Cordón, Body posture recognition by means of a genetic fuzzy finite state machine, IEEE 5th International Workshop on Genetic and Evolutionary Fuzzy Systems GEFS (2011), 60-65.

[7] E.N. Arcoverdo Neto, R.M. Duarte, R.M. Barreto, J.P. Magalhaes, C.M. Bastos, T.I. Ren and G.D.C. Cavalcanti, Enhanced real-time head pose estimation system for mobile device, Integrated Computer-Aided Engineering 21(3) (2014), 281-293.

[8] S.J. Brams and P.C. Fishburn, Going from theory to practice: The mixed success of approval voting, 2003 Handbook on Approval Voting Studies in Choice and Welfare Springer Verlag, (2010), 19-37.

[9] T.R. Burchfield and S. Venkatesan, Accelerometer-based human abnormal movement detection in wireless sensor networks, in: Proceedings of the 1st ACM SIGMOBILE International Workshop on Systems and Networking Support for Healthcare and Assisted Living Environments HealthNet'07 (2007), 67-69.
[10] J. Casillas, O. Cordón, M.J. del Jesus and F. Herrera, Genetic feature selection in a fuzzy rule-based classification system learning process, Information Sciences 136(1-4) (2001), 135157.

[11] C. Chira, J. Sedano, M. Camara, C. Prieto, J.R. Villar and E. Corchado, A cluster merging method for time series microarray with product values, International Journal of Neural Systems 24(6) (2014), 1450018-1/18.

[12] A.D. Dromerick and S.A. Khader, Medical complications during stroke rehabilitation, Advances in Neurology 92 (2003), 409-413.

[13] E. Duarte, B. Alonso, M.J. Fernández, J.M. Fernández, M. Flórez, I. García-Montes, J. Gentil, L. Hernández, F.J. Juan, J. Palomino, J. Vidal, E. Viosca, J.J. Aguilar, M. Bernabeu, I. Bori, F. Carrión, A. Déniz, I. Díaz, E. Fernández, P. Forastero, V. Iñigo, J. Junyent, N. Lizarraga, L. López de Munaín, I. Máñez, X. Miguéns, I. Sánchez and A. Soler, Stroke rehabilitation: care model, Rehabilitación 44(1) (2010), 60-68.

[14] S. Ghosh-Dastidar, H. Adeli and N. Dadmehr, Principal component analysis-enhanced cosine radial basis function neural network for robust epilepsy and seizure detection, IEEE Transactions on Biomedical Engineering 55(2) (2008), 512518.

[15] S. González, J.R. Villar, J. Sedano and C. Chira, A preliminary study on early diagnosis of illnesses based on activity disturbances, Proceedings of the 10th International Conference on Distributed Computing and Artificial Intelligence DCAI, Advances in Intelligent Systems and Computing 217 (2013), 521-527.

[16] H. Hemami, B.D. Clymer and M. Hemami, Simulation of control and synthesis of a constrained movement towards rehabilitation exercises, Integrated Computer-Aided Engineering 19(4) (2012), 351-364.

[17] L.J. Herrera, C. Fernandes, A.M. Mora, D. Migotina, R. Largo, A. Guillén and A.C. Rosa, Combination of heterogeneous EEG feature extraction methods and stacked sequential learning for sleep stage classification, International Journal of Neural Systems 23(3) (2013), 1350012-1/20.

[18] K. Hollands, Whole body coordination during turning while walking in stroke survivors, $\mathrm{Ph}$ D Thesis, School of Health and Population Sciences, University of Birmingham, 2010.

[19] C.S. Hogdson, To FAST or not to FAST, Stroke 38 (2007) 2631-2632.

[20] G. Iacca, F. Neri, E. Mininno, Y.-S. Ong and M.-H. Lim, Ockham's razor in memetic computing: Three stage optimal memetic exploration, Information Sciences 188(1) (2012), 17-43.

[21] A. Itrat, S. Alam, E. Walker and I. Katzan, Abstract TP180: Markedly elevated high-sensitive $C$-reactive protein (hsCRP) predicts early mortality among stroke patients, Stroke 44 (2013), ATP180.

[22] R. Kassab, J.-C. Lapayre, J.-B. Aupet, F. Marzani and C. Pieralli, Scars collaborative telediagnosis platform using adaptive image flow, Integrated Computer-Aided Engineering 20(1) (2013), 3-14.

[23] D. Li, L. Xu, E. Goodman, Y. Xu and Y. Wu, Integrating a statistical background-foreground extraction algorithm and SVM classifier for pedestrian detection and tracking, Integrated Computer-Aided Engineering 20(3) (2013), 201-216.

[24] J. Li, J. Liang, Q. Zhao, K. Hong and L. Zhang, Design of wheelchair assistive system directly steered by human thoughts, International Journal of Neural Systems 23(3) (2013), 1350013-1/12.

[25] J. Lin, E. Keogh, S. Lonardi and B. Chiu, A symbolic repre- 
sentation of time series, with implications for streaming algorithms, in: proceedings of the 8th ACM SIGMOD Workshop on Research Issues in Data Mining and Knowledge Discovery (2003).

[26] J. Lin, S. Williamson, K. Borne and D. DeBarr, Pattern recognition in time series, Advances in machine learning and data mining for astronomy, Chapman and Hall To Appear, A. Kamal, A. Srivastava, M. Way and J. Scargle, eds.

[27] Y. Liu and C. Pomalaza-Ráez, On-chip body posture detection for medical care applications using low-cost CMOS cameras, Integrated Computer-Aided Engineering 17(1) (2010), 3-13.

[28] R.J. Martis, U.R. Acharya, J.H. Tan. A. Petznick, C.K. Chua and E.Y.K. Ng, Application of intrinsic time-scale decomposition (ITD) to EEG signals for automated seizure prediction, International Journal of Neural Systems 23(5) (2013), 1350023-1/16.

[29] A. Meraoumia, S. Chitroub and A. Bouridane, 2D and 3D palmprint information, PCA and HMM for an improved person recognition performance, Integrated Computer-Aided Engineering 20(3) (2013), 303-319.

[30] M.P. Murray, A.B. Drought and R.C. Kory, Walking patterns of normal men, Journal of Bone and Joint Surgery 46(2) (1964), 335-360

[31] NHS UK, Stroke - Act F.A.S. T, http://www.nhs.uk/actfast/ Pages/stroke.aspx, technical report, National Health System, 2013.

[32] NSA, Warning Signs of Stroke, http://ww.stroke.org/site/Page Server?pagename $=\mathrm{SYMP}$, technical report, National Stroke Association, 2013.

[33] NSA, Reducing risk and recognizing symptoms, stroke is a brain attack! http://www.stroke.org/site/DocServer/ReducingRisk_8-09.pdf?docID $=3027$, technical report, National Stroke Association, 2013.

[34] I. Osorio, Automated seizure detection using EKG, International Journal of Neural Systems 24(2) (2014), 1450001-1/10.

[35] G. Rodriguez-Bermudeza, P.J. Garcia-Laencina and J. RocaDorda, Efficient automatic selection and combination of EEG features in least squares classifiers for motor-imagery brain computer interfaces, International Journal of Neural Systems 23(4) (2013), 1350015-1/17.

[36] S.H. Roy, M.S. Cheng, S.-S. Chang, J. Moore, G. de Luca, S.H. Nawab and C.J. de Luca, A combined sEMG and accelerometer system for monitoring functional activity in stroke, IEEE Transactions on Neural System Rehabilitation Engineering 17 (2009), 585-594.

[37] B. Sabayan, J. Gussekloo, W. de Ruijter, R.G.J. Westendorp and A.J.M. de Craen, Framingham stroke risk score and cognitive impairment for predicting first-time stroke in the oldest old, Stroke 44 (2013), 1866-1871.

[38] R.L. Sacco, S.E. Kasner, J.P. Broderick, L.R. Caplan, J.J. Connors, A. Culebras, M.S.V. Elkind, M.G. George, A.D. Hamdan, R.T. Higashida, B.L. Hoh, L.S. Janis, C.S. Kase, D.O. Kleindorfer, J.-M. Lee, M.E. Moseley, E.D. Peterson, T.N. Turan, A.L. Valderrama and H.V. Vinters, An updated definition of stroke for the 21 st century, A statement for healthcare professionals from the american heart association/ american stroke association, Stroke 44 (2013), 2064-2089.

[39] L. Sánchez and J. Otero, Boosting fuzzy rules in classification problems under single-winner inference, International Journal of Intelligent Systems 22(9) (2007), 1021-1035.

[40] Z. Sankari, H. Adeli and A. Adeli, Wavelet coherence model for diagnosis of alzheimer's disease, Clinical EEG and Neuroscience 43(3) (2012), 268-278.

[41] Y.E. Shao, C.-D. Hou and C.-C. Chiu, Hybrid intelligent modeling schemes for heart disease classification, Applied Soft Computing 14(A) (2014), 47-52.

[42] N. Siddique and H. Adeli, Computational intelligencesynergies of fuzzy logic, neural networks and evolutionary computing, Wiley, West Sussex, United Kingdom, 2012.

[43] J.B. Taylor, Jill bolte Taylor's stroke of insight, http://www. ted.com/talks/jill_bolte_taylor_s_powerful_stroke_of_insight .html, TED.com, 2008.

[44] S. Tolu, M. Vanegas, J.A. Garrido, N.R. Luque and E. Ros, Adaptive and predictive control of a simulated robot arm, International Journal of Neural Systems 23(3) (2013), 1350010$1 / 15$.

[45] J.R. Villar, S. González, J. Sedano, E. Corchado, L. Puigpinós and J. de Ciurana, Meta-heuristic improvements applied for steel sheet incremental cold shaping, Memetic Computing 4(4) (2012), 249-261.

[46] J.R. Villar, S. González, J. Sedano, C. Chira and J.M. Trejo, Human activity recognition and feature selection for stroke early diagnosis, in: 8th International Conference Hybrid Artificial Intelligent System HAIS 2013, LNCS 8073 (Springer Verlag) (2013), 659-668.

[47] J.R. Villar, S. González, J. Sedano, C. Chira and J.M. Trejo, Early diagnosis of Stroke: Bridging the gap through wearable sensors and computational models, Proceedings of the International Conference of Applied Mathematics ICAM9 (BaiaMare, Romania) (2013).

[48] J.R. Villar, C. Chira, S. González, J. Sedano and J.M. TrejoGabriel-Galán, Improving human activity recognition and its application in early stroke diagnosis, International Journal of Neural Systems, in press.

[49] X. Wang, Y. Dong, X. Qi, C. Huang and L. Hou, Cholesterol levels and risk of hemorrhagic stroke, A Systematic Review and Meta-Analysis, Stroke 44 (2013), 1833-1839.

[50] D. Wang, C. Quek and G. See Ng, Ovarian cancer diagnosis using a hybrid intelligent system with simple yet convincing rules, Applied Soft Computing 20 (2014), 25-39.

[51] A. Weingessel and K. Hornik, A robust subspace algorithm for principal component analysis, Int J Neur Syst 13(307) (2003), DOI: 10.1142/S0129065703001650.

[52] J.-Y. Yang, J.-S. Wang and Y.-P. Chen, Using acceleration measurements for activity recognition: An effective learning algorithm for constructing neural networks, Pattern Recognition Letters 29 (2008), 2213-2220.

[53] L. Zhang, T. Liu, S. Zhu and Z. Zhu, Human activity recognition based on triaxial accelerometer, 7th International Conference on Computing and Convergence Technology (ICCCT) (2012). 